\title{
Setting up a new home
}

Lymph nodes represent the first metastatic niche for many cancers and understanding how premetastatic conditioning occurs in these organs is crucial to prevent further colonization. Now, a study by Riedel et al. has proposed that fibroblastic reticular cells (FRCs), one component of the stroma, undergo dramatic transcriptional changes that could underlie the immunosuppressed state of tumour-draining lymph nodes (TDLNs).

To address how tumour-derived signals might influence the stromal landscape within TDLNs before metastasis, the authors used two independent mouse models of melanoma: a B16F10 allograft and a melanocytespecific Braf ${ }^{\mathrm{V} 600 \mathrm{E}}$ Pten $^{\text {lox }}$ model. In both mouse models the TDLNs were found to be considerably larger before the onset of metastasis when compared with lymph nodes from control mice. Coinciding with this enlargement of TDLNs was a substantial expansion of stromal populations of endothelial cells and FRCs.

FRCs support lymph node function by forming narrow conduits to control the passage of soluble molecules. Riedel and colleagues observed that an increased proliferation of FRCs within the TDLNs correlated with structural alterations in the FRC network, which included fewer branches and conduits with larger diameters and reduced size exclusion that enabled greater coverage of the LN by tumour-derived factors.
To assess whether the changing microenvironment within TDLNs might drive FRC adaptation, the authors used whole-genome transcriptional profiling of FRCs at early and late stages of lymph node transformation following tumour factor exposure. This approach revealed striking variation between the gene expression profiles of FRCs from TDLNs and those of control lymph nodes, and in particular that early-stage FRC reprogramming represented a discrete, intermediate state from that of the late stage. Interestingly, further characterization of FRCs in TDLNs demonstrated that this stromal cell type had upregulated expression of activation markers

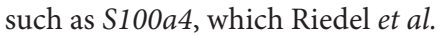
likened to the hyperactivated status of cancer-associated fibroblasts (CAFs) often found in the primary tumour microenvironment.

Another role of FRCs in maintaining lymph node homeostasis is to secrete numerous cues crucial for lymphocyte guidance and survival. Chemokine and cytokine signalling were among the various pathways deregulated in FRCs in TDLNs, with the expression of CC-chemokine ligand 21 (Ccl21) and interleukin 7 (Il7) considerably downregulated in these cells. Consistent with changes in FRC-associated homing cues, differences in the numbers and atypical localization of immune cell populations were detected; $\mathrm{CD} 4^{+}$ $\mathrm{T}$ cells were decreased while B cells, dendritic cells and macrophages were all increased within TDLNs at late stages.

This study reinforces the idea that the primary tumour communicates with lymph nodes to prime them as future sites of metastasis and highlights that tumour-derived signals can educate FRCs to remodel the lymph node stroma, potentially dampening immune responses, to establish a pro-tumorigenic niche.

Anna Dart

ORIGINAL ARTICLE Riedel, A. et al. Tumorinduced stromal reprogramming drives lymph node transformation. Nat. Immunol.

http://dx.doi.org/10.1038/ni.3492 (2016) 